\title{
Diversity and distribution of marine benthic algae and seagrasses in the tropical Kimberley, Western Australia
}

\author{
John M. Huisman 1*, Rainbo R.M. Dixon², Roberta A. Townsend' \\ and Gareth S. Belton² \\ 1 Western Australian Herbarium, Biodiversity and Conservation Science, Department of Biodiversity, \\ Conservation and Attractions, Locked Bag 104, Bentley Delivery Centre, Western Australia 6983, \\ Australia. \\ 2102 Hunters Rd, Basket Range, South Australia 5138, Australia. \\ * Corresponding author: john.huisman@dbca.wa.gov.au
}

\begin{abstract}
The algal and seagrass diversity at 177 marine survey stations in the Kimberley region, Western Australia (<30 m depth) was assessed, to establish species inventories and describe community structures. A total of 296 species of marine benthic algae and seagrasses were recorded, comprising 72 Chlorophyta (green algae), 4 Magnoliophyta (seagrasses), 35 Ochrophyta (brown algae) and 185 Rhodophyta (red algae). The generic diversity of the marine flora was largely typical for tropical Indo-Pacific coral reefs, but as the region was previously underexplored numerous new species were discovered. Records were grouped according to general location (Inshore, Midshelf, Ashmore and Hibernia Reefs, Rowley Shoals), with the greatest diversity recorded for Inshore (229 spp.), followed by the Rowley Shoals (102 spp.), Ashmore and Hibernia reefs (84 spp.), and Midshelf (40 spp.). Of the recorded taxa, 47\% (140 spp.) were exclusively found inshore, 1\% (3 spp.) exclusively Midshelf, 10\% (29 spp.) exclusively at the Rowley Shoals, and 5\% (16 spp.) exclusively at Ashmore and Hibernia reefs. Only 10 species were common to all general locations. Specimens collected during the survey formed the basis for the recognition of 52 new species and 4 taxonomic reassignments.
\end{abstract}

KEYWORDS: Indian Ocean, Kimberley Marine Bioregion, Algae, Seagrasses, Taxonomy, Woodside Collection Project

\section{INTRODUCTION}

Between 2009 and 2014, the Western Australian Museum undertook a series of baseline marine biota surveys in the Kimberley region of north-west Western Australia, with the aim of assessing the species diversity of a broad range of taxa, including fish, mobile invertebrates such as crustacea and echinoderms, and immobile invertebrates such as sponges and coral. As part of these surveys the marine flora (or 'seaweeds') were also collected and assessed, with the aim of establishing baseline data for the region and supplementing existing collections held in the Western Australian Herbarium. 'Flora' for the purposes of this contribution include the seagrasses, green algae and red algae (all Kingdom Plantae), plus the brown algae (Kingdom Chromista). The photosynthetic blue-green algae (Cyanobacteria), phytoplankton and mangroves were not assessed.
Historical collections of marine flora from the region were assessed by Huisman and Sampey (2014) and the value of establishing specimenbased inventories was described by Moore et al. (2020), particularly in relation to the present surveys. A general introduction to the surveys, plus descriptions of broader methodology were described by Bryce et al. $(2018 a, b)$ and will not be repeated here.

\section{AIMS}

The aim of this study was to record the biodiversity of the shallow water $(<30 \mathrm{~m})$ algal and seagrass flora in the Kimberley region of Western Australia over a wide range of habitats and locations.

\section{MATERIALS AND METHODS}

The survey locations and general field methods are described in detail in Bryce et al. (2018a) and 
Bryce et al. (2018b). Algal and seagrass collections were made by intertidal reef walks or subtidal SCUBA dives covering a range of depths to $30 \mathrm{~m}$. Specimens were pressed while fresh, with small portions dried in silica gel for DNA analysis or $5 \%$ formalin/seawater for later microscopic examination, or in the case of encrusting coralline algae washed and air-dried.

As these surveys often represented the first opportunities to collect marine flora at remote Kimberley locations, it was decided to maximise biodiversity by collecting at a range of depths, rather than along a transect at a single depth. As was noted for octocorals (Bryce et al. 2018b), overall biodiversity was always higher than transect biodiversity, and this would also be expected for the flora.

Collections made during the six expeditions were processed as herbarium vouchers and lodged in the Western Australian Herbarium (PERTH) and collection details entered into WAHERB, the specimen database of the WA Herbarium. Collections were made by Huisman (2010, 2011, 2013), Dixon $(2009,2012)$ and Belton (2014). A total of 852 records were added. These primarily represent the larger macroalgae that were suitable for pressing or boxing. Smaller filamentous species were prepared as microscope slides; where these represented new taxa they have been added to the PERTH database, but many slide-mounted specimens are yet to be incorporated. Additional records have been drawn from Huisman et al. (2009) for the Rowley Shoals and Huisman (2015, 2018). Results of these and earlier surveys of the marine algae of tropical Western Australia are presented in two volumes of the 'Algae of Australia' series (Huisman 2015, 2018) and images of the larger algae are included in Huisman (2019).

There is a fundamental problem in assessing biogeography based on these collections of algae. Many species are small and filamentous, often epiphytic, and cannot be identified reliably in the field. The recording of epiphytic species is generally undertaken during post-expedition examination of larger taxa and as such is essentially serendipitous. Thus, there is a high potential for bias based on incomplete taxon sampling, with the smaller epiphytes going unnoticed and thus not recorded. Similarly, crustose species (such as encrusting coralline algae and the Peyssonneliaceae) are difficult to collect and identify. Many of the species in these groups are essentially cryptic and not distinguishable in the field, and in most cases the collections were identified based on post-expedition DNA sequence analyses (for example see Dixon 2018). It is highly unlikely that the full complement of crustose species was collected from each locality and as such records of these species are also potentially biased.

\section{RESULTS}

A total of 296 species of marine benthic algae and seagrasses are recorded herein (Table 1). These comprise 72 Chlorophyta (green algae), 4 Magnoliophyta (seagrasses), 35 Ochrophyta (brown algae) and 185 Rhodophyta (red algae). Here, as in Bryce et al. (2018b), the collection sites are divided into four categories and the species present are listed in Table 1. Examples of species recorded are presented in Figures 1-3. The greatest species diversity (216 spp.) was recorded for the Kimberley Inshore, with lower diversities at the Rowley Shoals (81 spp.), Ashmore and Hibernia Reefs (62 spp.) and the Midshelf (31 spp.). This trend undoubtedly reflects a greater diversity of Inshore habitats, but any conclusions must be tempered by the caveats concerning recording bias indicated earlier, plus the differences in collection effort. Of the 179 sites visited, 110 were Inshore, 57 were offshore (Ashmore and Hibernia Reefs, Rowley Shoals) and only 12 were Midshelf.

TABLE 1 Species of algae and seagrasses recorded from the Kimberley survey area. Names in bold are species newly described or revised taxonomically based on specimens collected during these surveys.

\begin{tabular}{|c|c|c|c|c|}
\hline Таха & $\begin{array}{l}\text { Kimberley } \\
\text { Inshore }\end{array}$ & Midshelf & $\begin{array}{l}\text { Ashmore } \\
\text { and Hibernia } \\
\text { Reefs }\end{array}$ & $\begin{array}{l}\text { Rowley } \\
\text { Shoals }\end{array}$ \\
\hline
\end{tabular}

\section{CHLOROPHYTA}

Family: Anadyomenaceae

Anadyomene plicata C. Agardh, 1823

1

1

Anadyomene wrightii Harv. ex J.E. Gray, 1866

Family: Boodleaceae

Boodlea composita (Harv.) Brand, 1904

1

Boodlea vanbosseae Reinbold, 1905

1

1

1

1 


\begin{tabular}{|c|c|c|c|c|}
\hline Таха & $\begin{array}{l}\text { Kimberley } \\
\text { Inshore }\end{array}$ & Midshelf & $\begin{array}{l}\text { Ashmore } \\
\text { and Hibernia } \\
\text { Reefs }\end{array}$ & $\begin{array}{l}\text { Rowley } \\
\text { Shoals }\end{array}$ \\
\hline
\end{tabular}

Cladophoropsis vaucheriiformis (Aresch.) Papenf., 1958

Phyllodictyon anastomosans (Harv.) Kraft \& M.J. Wynne, 1996

Phyllodictyon orientale (A. Gepp \& E. Gepp)

Kraft \& M.J. Wynne, 1996

Family: Bryopsidaceae

Bryopsis pennata J.V. Lamour., 1809

Family: Caulerpaceae

Caulerpa agardhii Weber Bosse, 1898

Caulerpa chemnitzia (Esper) J.V. Lamour., 1809

Caulerpa corynephora Mont., 1842

Caulerpa cupressoides (Vahl) C. Agardh, 1817

Caulerpa cylindracea Sond., 1845

Caulerpa filicoides Yamada, 1936

Caulerpa lamourouxii (Turner) C. Agardh, 1817

Caulerpa lentillifera J. Agardh, 1837

Caulerpa macrodisca Decne., 1842

Caulerpa racemosa (Forssk.) J. Agardh, 1873

Caulerpa serrulata (Forssk.) J. Agardh, 1837

Caulerpa sertularioides (S.G. Gmelin) M. Howe, 1905

Caulerpa taxifolia (Vahl) C. Agardh, 1817

Caulerpa urvilleana Mont., 1845

Caulerpa verticillata J. Agardh, 1847

Caulerpa webbiana Mont., 1837

Family: Cladophoraceae

Chaetomorpha indica (Kütz.) Kütz., 1849

Cladophora catenata Kütz., 1843

Cladophora sp.

Lychaete herpestica (Mont.) M.J. Wynne, 2017

\section{Family: Codiaceae}

Codium arabicum Kütz., 1856

Codium dwarkense Børgesen, 1947

Codium geppiorum O.C. Schmidt, 1923

Codium strangulatum Chacana \& P.C. Silva, 2015

\section{Family: Dasycladaceae}

Bornetella oligospora Solms., 1892

Neomeris bilimbata Koster, 1937

Parvocaulis parvulus (Solms) S. Berger, Fettweiss,

Gleissberg, L.B. Liddle, U. Richt., Sawitzky

\& Zuccarello, 2003

\section{Family: Derbesiaceae}

Derbesia attenuata E.Y. Dawson, 1954

\section{Family: Dichotomosiphonaceae}

Avrainvillea amadelpha (Mont.) A. Gepp \& E. Gepp, 1908

Avrainvillea erecta (Berk.) A. Gepp \& E. Gepp, 1911 


\begin{tabular}{|c|c|c|c|c|}
\hline Taxa & $\begin{array}{l}\text { Kimberley } \\
\text { Inshore }\end{array}$ & Midshelf & $\begin{array}{l}\text { Ashmore } \\
\text { and Hibernia } \\
\text { Reefs }\end{array}$ & $\begin{array}{l}\text { Rowley } \\
\text { Shoals }\end{array}$ \\
\hline
\end{tabular}

Family: Dictyosphaeriaceae

Dictyosphaeria cavernosa (Forssk.) Børgesen, 1932

Dictyosphaeria versluysii Weber Bosse, 1905

Valoniopsis pachynema (G. Martens) Børgesen, 1934

\section{Family: Halimedaceae}

Halimeda cylindracea Decne., 1842

Halimeda discoidea Decne., 1842

Halimeda distorta (Yamada) Hillis-Col., 1968

Halimeda gigas W.R. Taylor, 1950

Halimeda gracilis J. Agardh, 1887

Halimeda heteromorpha N’Yeurt, 2006

Halimeda lacunalis W.R. Taylor, 1950

Halimeda macroloba Decne., 1841

Halimeda macrophysa Askenasy, 1888

Halimeda micronesica Yamada, 1941

Halimeda minima (W.R. Taylor) Hillis-Col., 1968

Halimeda opuntia (L.) J.V. Lamour., 1816

Halimeda taenicola W.R. Taylor, 1950

Halimeda tuna (J. Ellis \& Sol.) J.V. Lamour., 1816

Halimeda velasquezii W.R. Taylor, 1962

Halimeda xishaensis C.K. Tseng \& M.L. Dong, 1980

\section{Family: Rhipiliaceae}

Rhipidosiphon javensis Mont., 1842

Rhipilia nigrescens Coppejans \& Prud'homme, 1990

Rhipiliopsis echinocaulos (Cribb) Farghaly, 1986

Rhipiliopsis papuensis Coppejans, De Clerck \& Leliaert, 1999

Family: Siphonocladaceae

Boergesenia forbesii (Harv.) Feldmann, 1938

\section{Family: Udoteaceae}

Tydemania expeditionis Weber Bosse, 1901

Udotea argentea Zanardini, 1858

Udotea flabellum (J. Ellis \& Sol.) M. Howe, 1904

Udotea glaucescens J. Agardh, 1887

Udotea orientalis A. Gepp \& E. Gepp, 1911

\section{Family: Ulvaceae}

Ulva flexuosa Wulfen, 1803

Ulva kraftiorum Huisman, 2015

\section{Family: Ulvellaceae}

Ulvella lens P. Crouan \& H. Crouan, 1859

\section{Family: Valoniaceae}

Valonia fastigiata J. Agardh, 1887

Valonia ventricosa J. Agardh, 1887 


\begin{tabular}{|c|c|c|c|c|}
\hline Таха & $\begin{array}{l}\text { Kimberley } \\
\text { Inshore }\end{array}$ & Midshelf & $\begin{array}{l}\text { Ashmore } \\
\text { and Hibernia } \\
\text { Reefs }\end{array}$ & $\begin{array}{l}\text { Rowley } \\
\text { Shoals }\end{array}$ \\
\hline
\end{tabular}

\section{MAGNOLIOPHYTA}

Family: Cymodoceaceae

Thalassodendron ciliatum (Forssk.) Hartog, 1970

Family: Hydrocharitaceae

Halophila decipiens Ostenf., 1902

Halophila ovalis (R. Br.) Hook.f., 1858

Thalassia hemprichii (Ehrenb.) Asch., 1871

\section{OCHROPHYTA}

\section{Family: Acinetosporaceae}

Feldmannia indica (Sond.) Womersley \& A. Bailey, $1970+$

Feldmannia irregularis (Kütz.) Hamel, 1939

\section{Family: Chordariaceae}

Nemacystus decipiens (Suringar) Kuck., 1929

\section{Family: Dictyotaceae}

Canistrocarpus cervicornis (Kütz.) De Paula \& De Clerck, $2006+$ Dictyopteris australis (Sond.) Askenasy, 1888

Dictyopteris repens (Okamura) Børgesen, 1924

Dictyopteris woodwardia (Turner) C. Agardh, 1817

Dictyota ciliolata Kütz., 1859

Dictyota friabilis Setch., 1926

Dictyota sp.

Lobophora variegata (J.V. Lamour.) Oliviera, 1977

Padina australis Hauck, 1997

Padina boryana Thivy in W.R. Taylor, 1966

Padina sp.

Spatoglossum macrodontum J. Agardh, 1882

Stypopodium flabelliforme Weber Bosse, 1913

\section{Family: Sargassaceae}

Hormophysa cuneiformis (J.F. Gmel.) P.C. Silva, 1987

Sargassopsis decurrens (Turner) Trevis., 1843

Sargassum aquifolium (Turner) C. Agardh, 1820

Sargassum ilicifolium (Turner) C. Agardh, 1820

Sargassum ligulatum C. Agardh, 1824

Sargassum linearifolium (Turner) C. Agardh, 1820

Sargassum marginatum (C. Agardh) J. Agardh, 1848

Sargassum polycystum C. Agardh, 1824

Sargassum rasta R.R.M. Dixon \& Huisman, 2015

Sargassum swartzii C. Agardh, 1820

Sirophysalis trinodis (Forssk.) Kütz., 1849

Turbinaria conoides (J. Agardh) Kütz., 1860

Turbinaria gracilis Sond., 1845

Turbinaria ornata (Turner) J. Agardh, 1848 


\begin{tabular}{|c|c|c|c|c|}
\hline Taxa & $\begin{array}{l}\text { Kimberley } \\
\text { Inshore }\end{array}$ & Midshelf & $\begin{array}{l}\text { Ashmore } \\
\text { and Hibernia } \\
\text { Reefs }\end{array}$ & $\begin{array}{l}\text { Rowley } \\
\text { Shoals }\end{array}$ \\
\hline
\end{tabular}

Family: Scytosiphonaceae

Colpomenia sinuosa (Roth) Derbès \& Solier, 1851

Hydroclathrus clathratus (C. Agardh) Howe, 1920

Rosenvingea intricata (J. Agardh) Børgesen, 1914

Family: Sphacelariaceae

Sphacelaria novae-hollandiae Sond., 1845

Sphacelaria rigidula Kütz., 1843

\section{RHODOPHYTA}

\section{Family: Acrochaetiaceae}

Acrochaetium liagorae Børgesen, 1915

\section{Family: Acrosymphytaceae}

Acrosymphyton taylorii I.A. Abbott, 1962

Family: Bonnemaisoniaceae

Asparagopsis taxiformis (Delile) Trevisan, 1845

Family: Callithamniaceae

Aglaothamnion cordatum (Børgesen) Feldm.-Maz., 1941

Crouania dampieriana Huisman, 2018

Crouania eliseae C.W. Schneider, 2004

Seirospora orientalis Kraft, 1988

\section{Family: Caulacanthaceae}

Pseudocaulacanthus spinescens Huisman, 2018

\section{Family: Ceramiaceae}

Antithamnionella graeffei (Grunow) Athanas., 1996

Balliella crouanioides (Itono) Itono \& Tak. Tanaka, 1973

Balliella subcorticata Itono \& Tak. Tanaka, 2018

Centroceras gasparrinii (Menegh.) Kütz., 1849

Ceramium aduncum Y. Nakam., 1950

Ceramium affine Setch. \& N.L. Gardner, 1930

Ceramium cingulatum Weber Bosse, 1923

Ceramium codii (H. Richards) Feldm.-Maz., 1938

Ceramium isogonum Harv., 1855

Ceramium macilentum J. Agardh, 1894

Ceramium phillipsiae Huisman, 2018

Ceramium pseudocodii Huisman, 2018

Ceramium subdichotomum Weber Bosse, 1923

Corallophila kleiwegii Weber Bosse, 1923

Gayliella fimbriata (Setch. \& N.L. Gardner)

T.O. Cho \& S.M. Boo, 2008

Gayliella macrotricha (Feldm.-Maz.) Huisman, 2018

Gayliella transversalis (Collins \& Herv.)

T.O. Cho \& Fredericq, 2008 


\begin{tabular}{|c|c|c|c|c|}
\hline Таха & $\begin{array}{l}\text { Kimberley } \\
\text { Inshore }\end{array}$ & Midshelf & $\begin{array}{l}\text { Ashmore } \\
\text { and Hibernia } \\
\text { Reefs }\end{array}$ & $\begin{array}{l}\text { Rowley } \\
\text { Shoals }\end{array}$ \\
\hline
\end{tabular}

\section{Family: Champiaceae}

Champia pseudoparvula Huisman \& G.W. Saunders, 2018

Champia stipitata Huisman, 2000

Champia subcompressa Huisman \& G.W. Saunders, 2018

Coelothrix irregularis (Harv.) Børgesen, 1920

\section{Family: Corallinaceae}

Jania pedunculata J.V. Lamour., 1816

Jania spectabilis (Grunow) J.H. Kim, Guiry \& H.G. Choi, 2007

\section{Family: Corynocystaceae}

Corynocystis prostrata Kraft, 1999

Family: Corynomorphaceae

Corynomorpha prismatica (J. Agardh) J. Agardh, 1876

Family: Cystocloniaceae

Hypnea charoides J.V. Lamour., 1813

Hypnea pannosa J. Agardh, 1847

\section{Family: Dasyaceae}

Dasya frutescens Harv., 1855

Dasya sp.

Heterosiphonia crassipes (Harv.) Falkenb., 1901

\section{Family: Delesseriaceae}

Hypoglossum anomalum M.J. Wynne \& D.L. Ballant., 1986 + Hypoglossum caloglossoides M.J. Wynne \& Kraft, $1985+$

Martensia millarii Huisman, 2018

Vanvoorstia spectabilis Harv., 1854

Zellera tawallina G. Martens, 1866

\section{Family: Dumontiaceae}

Dudresnaya hawaiiensis R.K.S. Lee, 1963

Gibsmithia indopacifica D. Gabriel, Draisma \& Fredericq, 2017

Gibsmithia larkumii Kraft, 1986

Family: Etheliaceae

Ethelia denizotii K.R. Dixon, 2015

\section{Family: Faucheaceae}

Gloioderma iyoense Okamura, 1934

\section{Family: Galaxauraceae}

Actinotrichia coccinea

Huisman, G.H. Boo \& S.M. Boo, 2018

Actinotrichia fragilis (Forssk.) Børgesen, 1932

Dichotomaria sibogae (Weber Bosse)

Huisman, G.H. Boo, S.M. Boo \& S.-M. Lin, 2018

Galaxaura elongata J. Agardh, 1876

Galaxaura glabriuscula Kjellm., 1900

Galaxaura indica Huisman, G.H. Boo \& S.M. Boo, 2018 


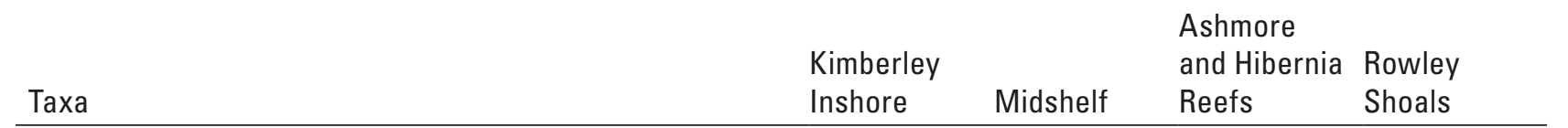

Galaxaura pacifica Tanaka, 1935

Tricleocarpa fastigiata (Decne.)

Huisman, G.H. Boo \& S.M. Boo, 2018

Family: Gelidiaceae

Pterocladiella caerulescens (Kütz.) Santel. \& Hommers., 1997

Family: Gelidiellaceae

Gelidiella acerosa (Forssk.) Feldmann \& Hamel, 1934

Gelidiella fanii S.-M. Lin, 2008

Family: Gracilariaceae

Gracilaria canaliculata Sond., 1871

Gracilaria salicornia (C. Agardh) E.Y. Dawson, 1954

Gracilaria vieillardii P.C. Silva, 1987

Gracilaria webervanbosseae

Huisman \& G.W. Saunders, 2018

Hydropuntia urvillei Mont., 1842

Family: Halymeniaceae

Halymenia maculata J. Agardh, 1885

Halymenia malaysiana

P.L. Tan, P.E. Lim, S.-M. Lin \& Phang, 2018

Halymenia villosa Huisman \& De Clerck, 2018

Family: Hapalidiaceae

Lithothamnion proliferum Foslie, 1904

Lithothamnion sonderi Hauck, 1883

Melobesia tomitaroi Kloczkova, 1987

Mesophyllum cf. funafutiense (Foslie) Verheij, 1993

Rhizolamellia colli Shevejko, 1982

Family: Hydrolithaceae

Hydrolithon farinosum (J.V. Lamour.)

Penrose \& Y.M. Chamb., 1993

Hydrolithon boergesenii (Foslie) Foslie, 1909

Family: Hymenocladiaceae

Asteromenia exanimans

G.W. Saunders, C.E. Lane, C.W. Schneid. \& Kraft, 2006

Asteromenia kimberleyensis

Huisman \& G.W. Saunders, 2018

Asteromenia poeciloderma

Huisman \& G.W. Saunders, 2018

Family: Kallymeniaceae

Leiomenia imbricata Huisman \& G.W. Saunders, 2017

Leiomenia lacunata Huisman \& G.W. Saunders, 2017

Rhytimenia maculata (Weber Bosse)

Huisman \& G.W. Saunders, 2016

Family: Liagoraceae

Dotyophycus damaru Huisman \& S.-M. Lin, 2018

Ganonema farinosum (J.V.Lamour.)

K.C. Fan \& Yung C. Wang, 1974 


Taxa

Ganonema robustum Huisman \& S.-M. Lin, 2018

Hommersandiophycus kraftii Huisman \& S.-M. Lin, 2018

Izziella vulcanensis Huisman \& S.-M. Lin, 2018

Liagora ceranoides J.V. Lamour., 1816

Macrocarpus perennis (I.A.Abbott)

S.-M. Lin, S.Y. Yang \& Huisman, 2018

Neoizziella asiatica S.-M. Lin, S.Y. Yang \& Huisman, 2011

Titanophycus saundersii Huisman \& S.-M. Lin, 2018

Trichogloea requienii (Mont.) Kütz., 1847

Yoshizakia indopacifica S.-M. Lin, Huisman \& Payri, 2013

Family: Lithophyllaceae

Amphiroa crassa J.V. Lamour., 1824

Amphiroa foliacea J.V. Lamour., 1824

Amphiroa fragilissima (L.) J.V. Lamour., 1816

Amphiroa tribulus (J. Ellis \& Sol.) J.V. Lamour., 1816

Lithophyllum kaiseri (Heydr.) Heydr., 1897

Lithophyllum longense

Hern.-Kant., P.W. Gabrielson \& R.A. Towns., 2018

Titanoderma pustulatum (J.V. Lamour.) Nägeli, 1858

Family: Lomentariaceae

Ceratodictyon intricatum (C. Agardh) R.E. Norris, 1987

Ceratodictyon scoparium (Mont. \& Millardet) R.E. Norris, 1987

Ceratodictyon spongiosum Zanardini, 1878

Family: Mastophoraceae

Mastophora multistrata Keats, 2009

Mastophora rosea (C. Agardh) Setch., 1943

Family: Nemastomataceae

Predaea laciniosa Kraft, 1984

Predaea sophieae Huisman, 2018

Predaea weldii Kraft \& I.A. Abbott, 1971

Family: Peyssonneliaceae

Incendia cryptica K.R. Dixon, 2018

Incendia homosorora K.R. Dixon, 2018

Incendia undulata K.R. Dixon, 2013

Peyssonnelia atricolor K.R. Dixon, 2018

Peyssonnelia clathrata K.R.Dixon, 2018

Peyssonnelia confluens K.R. Dixon, 2018

Peyssonnelia impermia K.R. Dixon, 2018

Peyssonnelia pseudostrata K.R. Dixon, 2018

Peyssonnelia rainboae K.R. Dixon, 2018

Peyssonnelia tenuiderma K.R. Dixon, 2018

Peyssonnelia webervanbosseae K.R. Dixon, 2018

Polystrata erupta K.R. Dixon, 2018

Polystrata kimberleyensis K.R. Dixon, 2018

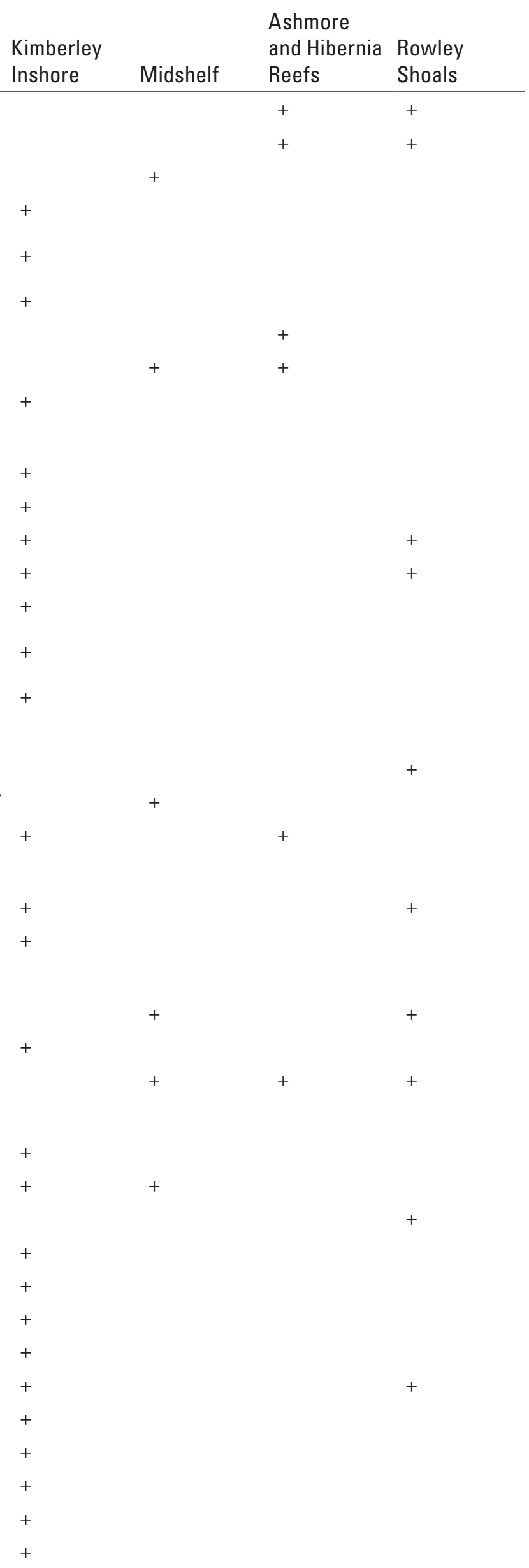




\begin{tabular}{|c|c|c|c|c|}
\hline Таха & $\begin{array}{l}\text { Kimberley } \\
\text { Inshore }\end{array}$ & Midshelf & $\begin{array}{l}\text { Ashmore } \\
\text { and Hibernia } \\
\text { Reefs }\end{array}$ & $\begin{array}{l}\text { Rowley } \\
\text { Shoals }\end{array}$ \\
\hline
\end{tabular}

Ramicrusta appressa K.R. Dixon, 2013

Ramicrusta melanoidea K.R. Dixon, 2018

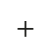

Seiria magnifusa K.R. Dixon, 2018

Sonderophycus fervens K.R. Dixon, 2018

\section{Family: Porolithaceae}

Dawsoniolithon conicum (E.Y. Dawson)

Caragnano, Foetisch, Maneveldt \& Payri, 2018

Dawsoniolithon gabrielsonii R.A. Towns. \& Huisman, 2018

Floiophycus walteradeyi R.A. Towns. \& Huisman, 2018

Porolithon imitatum R.A. Towns. \& G.W. Saunders, 2018

Porolithon maneveldtii R.A. Towns. \& Huisman, 2018

Porolithon pachydermum (Foslie) Foslie, 1909

Porolithon penroseae R.A. Towns. \& P.W. Gabrielson, 2018

\section{Family: Rhizophyllidaceae}

Contarinia pacifica (Børgesen) Denizot, 1968

Contarinia ventriglandis K.R. Dixon, 2018

Portieria hornemannii (Lyngb.) P.C. Silva, 1987

\section{Family: Rhodomelaceae}

Acanthophora ramulosa Kütz., 1843

Acanthophora spicifera (Vahl) Børgesen, 1910

Amansia rhodantha (Harv.) J. Agardh, 1841

Bostrychia tenella (J.V. Lamour.) J. Agardh, 1863

Chondria armata (Kütz.) Okamura, 1907

Chondria dangeardii E.Y. Dawson, 1954

Chondria minutula Weber Bosse, 1923

Chondria transversalis Børgesen, 1938

Digenea simplex (Wulfen) C. Agardh, 1822

Herposiphonia elongata M. Masuda \& K. Kogame, 2000

Herposiphonia mermaidensis Huisman, 2018

Laurencia brongniartii J. Agardh, 1841

Laurencia dendroidea J. Agardh, 1852

Laurencia similis K.W. Nam \& Y. Saito, 1991

Leveillea jungermannioides (E. Hering \& G. Martens)

Harv., 1855

Lophocladia sp.

Melanothamnus ferulaceus (J. Agardh)

Díaz-Tapia \& Maggs, 2017

Melanothamnus quadratus (Hollenb.) Huisman, 2018

Melanothamnus upolensis (Grunow) Díaz-Tapia \& Maggs, 2017

Palisada perforata (Bory) K.W. Nam, 2007

Polysiphonia beltoniorum Huisman, 2018

Polysiphonia kimiae Huisman, 2018

Spirocladia loochooensis (Yendo) T. Yoshida, 1989

Tolypiocladia calodictyon (Kütz.) P.C. Silva, 1952
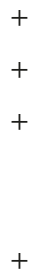


\begin{tabular}{|c|c|c|c|c|}
\hline Taxa & $\begin{array}{l}\text { Kimberley } \\
\text { Inshore }\end{array}$ & Midshelf & $\begin{array}{l}\text { Ashmore } \\
\text { and Hibernia } \\
\text { Reefs }\end{array}$ & $\begin{array}{l}\text { Rowley } \\
\text { Shoals }\end{array}$ \\
\hline Tolypiocladia glomerulata (C. Agardh) F. Schmitz, 1897 & + & & + & \\
\hline Vidalia melvillii (J. Agardh) F. Schmitz, 1895 & + & & & \\
\hline \multicolumn{5}{|l|}{ Family: Rhodymeniaceae } \\
\hline Botryocladia leptopoda (J. Agardh) Kylin, 1931 & + & & & \\
\hline Botryocladia skottsbergii (Børgesen) Levring, 1943 & & + & + & \\
\hline Chamaebotrys boergesenii (Weber Bosse) Huisman, 1996 & + & & & + \\
\hline Coelarthrum opuntia (Endl.) Børgesen, 1937 & + & & & \\
\hline \multicolumn{5}{|l|}{ Family: Schizymeniaceae } \\
\hline Titanophora pikeana (Dickie) Feldmann, 1942 & & & + & \\
\hline \multicolumn{5}{|l|}{ Family: Scinaiaceae } \\
\hline Scinaia tsinglanensis C.K. Tseng, 1941 & + & + & & \\
\hline \multicolumn{5}{|l|}{ Family: Sebdeniaceae } \\
\hline Cryptocallis dixoniorum Huisman \& G.W. Saunders, 2018 & + & & & \\
\hline Sebdenia polydactyla (Børgesen) M.P. Balakr., 1961 & + & & & \\
\hline \multicolumn{5}{|l|}{ Family: Solieriaceae } \\
\hline Betaphycus speciosus (Sond.) P.C. Silva, 1996 & + & & & \\
\hline Eucheuma arnoldii Weber Bosse, 1928 & + & & + & \\
\hline Eucheuma denticulatum (Burm.f.) Collins \& Herv., 1917 & + & & & \\
\hline Solieria robusta (Grev.) Kylin, 1932 & + & & & \\
\hline \multicolumn{5}{|l|}{ Family: Spongitaceae } \\
\hline $\begin{array}{l}\text { Neogoniolithon brassica-florida (Harv.) } \\
\text { Setch. \& L.R. Mason, } 1943\end{array}$ & + & & & + \\
\hline \multicolumn{5}{|l|}{ Family: Sporolithaceae } \\
\hline $\begin{array}{l}\text { Sporolithon episoredion (W.H. Adey, R.A. Towns. \& Boykins) } \\
\text { Verheij, } 1992\end{array}$ & & & & + \\
\hline \multicolumn{5}{|l|}{ Family: Spyridiaceae } \\
\hline Spyridia filamentosa (Wulfen) Harv., 1833 & + & & & + \\
\hline \multicolumn{5}{|l|}{ Family: Stylonemataceae } \\
\hline Stylonema alsidii (Zanardini) K.M. Drew, 1956 & & & + & \\
\hline \multicolumn{5}{|l|}{ Family: Tsengiaceae } \\
\hline Tsengia flammea Huisman \& G.W. Saunders, 2018 & & & + & \\
\hline \multicolumn{5}{|l|}{ Family: Wrangeliaceae } \\
\hline Anotrichium tenue (C. Agardh) Nägeli, 1862 & + & & + & + \\
\hline Grallatoria reptans M. Howe, 1920 & + & & & \\
\hline Griffithsia heteromorpha Kütz., 1863 & + & & & \\
\hline Griffithsia schousboei Mont., 1839 & + & & & \\
\hline Haloplegma duperreyi Mont., 1842 & & & + & + \\
\hline Spongoclonium caribaeum (Børgesen) M.J. Wynne, 2005 & + & & & \\
\hline Wrangelia sp. & + & + & + & \\
\hline \multicolumn{5}{|l|}{ Family: Yamadaellaceae } \\
\hline Yamadaella australis Huisman \& S.-M. Lin, 2018 & + & & & \\
\hline Yamadaella caenomyce (Decne.) I.A. Abbott, 1970 & + & & & \\
\hline
\end{tabular}



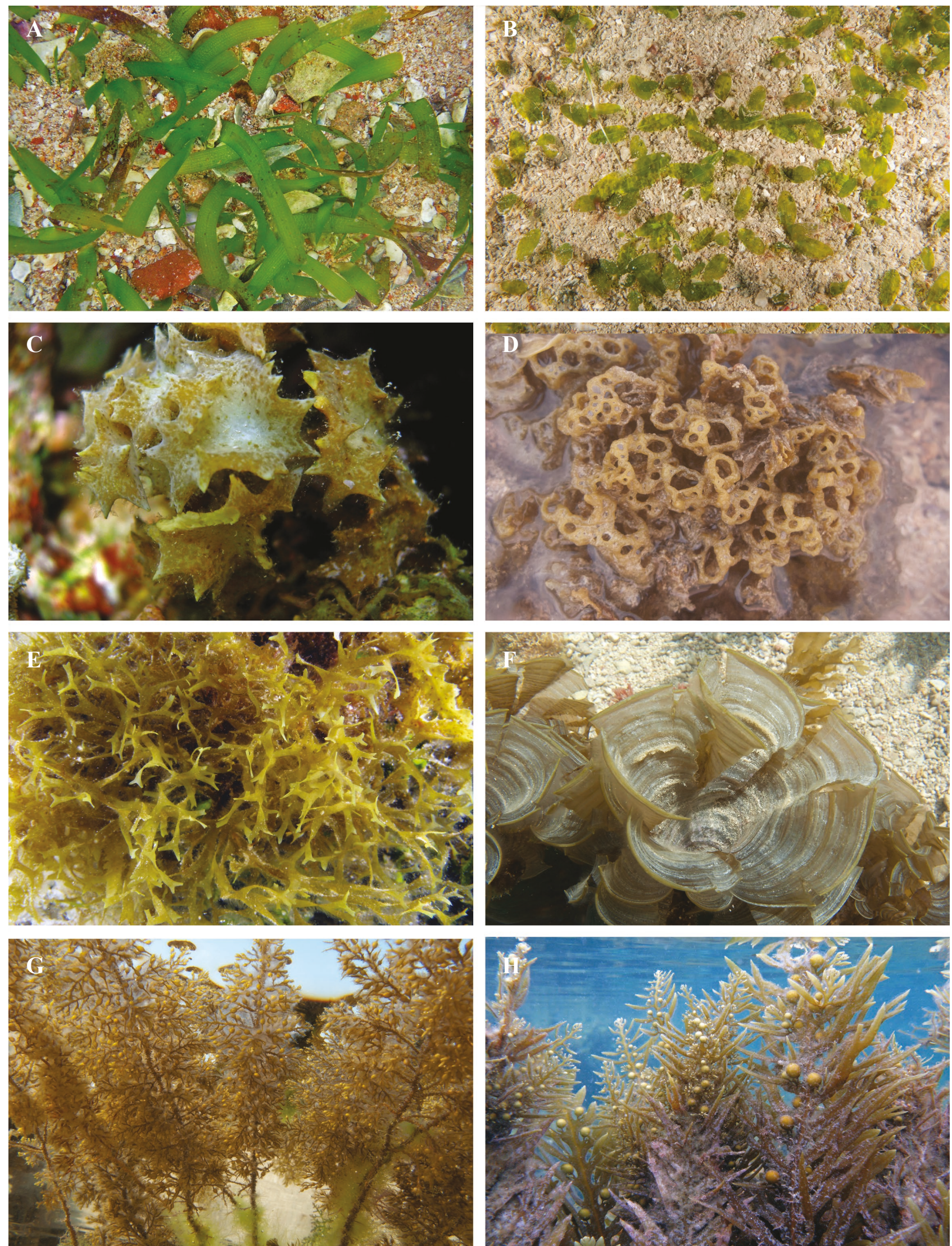

FIGURE 1 Species of seagrasses and brown algae from the Kimberley survey area: A) Thalassia hemprichii (Hydrocharitaceae); B) Halophila decipiens (Hydrocharitaceae); C) Turbinaria ornata (Sargassaceae); D) Hydroclathrus clathratus (Scytosiphonaceae); E) Canistrocarpus cervicornis (Dictyotaceae); F) Padina australis (Dictyotaceae); G) Sirophysalis trinodis (Sargassaceae); H) Sargasssopsis decurrens (Sargassaceae). 

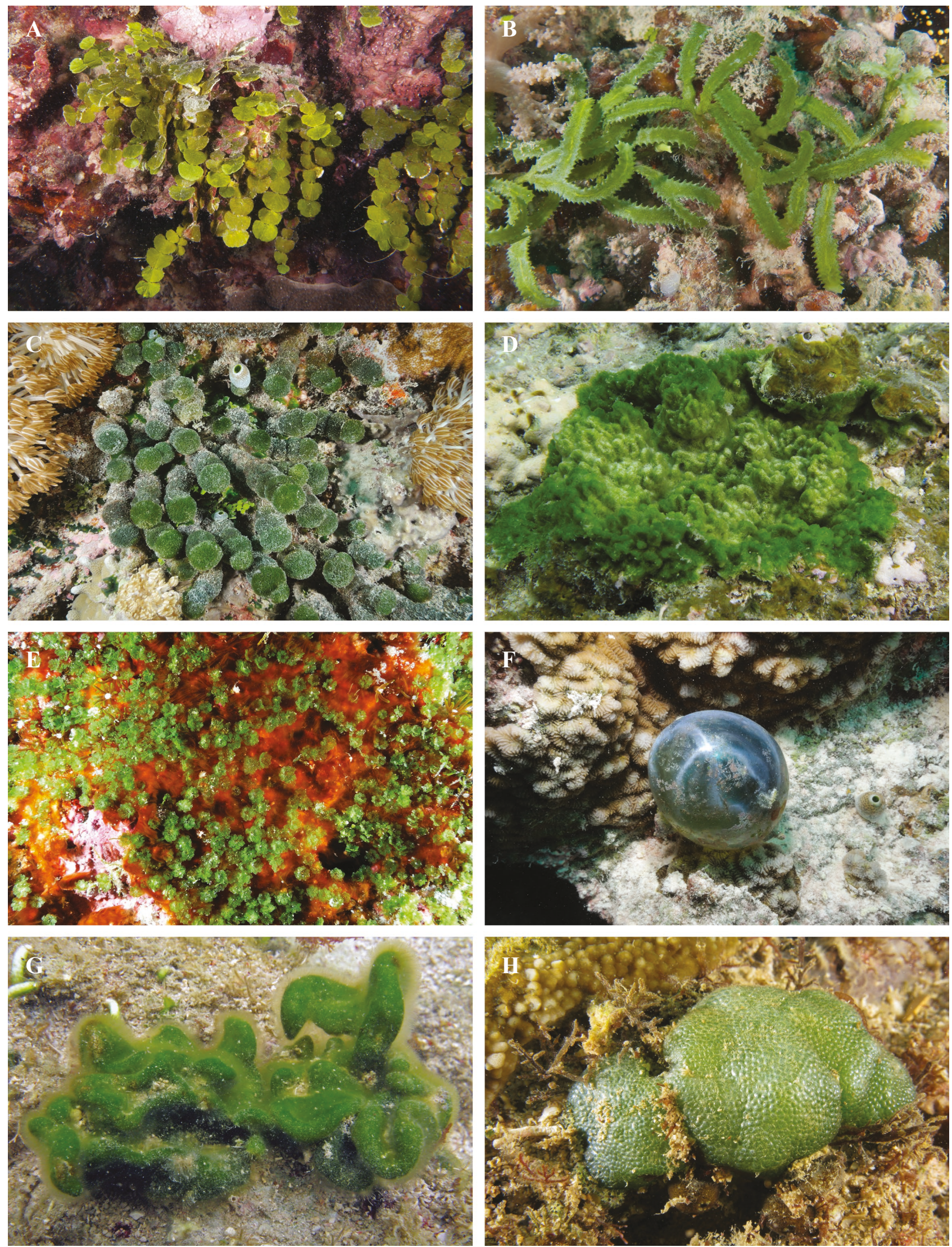

FIGURE 2 Species of green algae from the Kimberley survey area: A) Halimeda minima (Halimedaceae); B) Caulerpa serrulata (Caulerpaceae); C) Tydemania expeditionis (Udoteaceae); D) Cladophoropsis vaucheriiformis (Boodleaceae); E) Caulerpa filicoides (Caulerpaceae); F) Valonia ventricosa (Valoniaceae); G) Codium arabicum (Codiaceae); H) Dictyosphaeria versluysii (Dictyosphaeriaceae). 

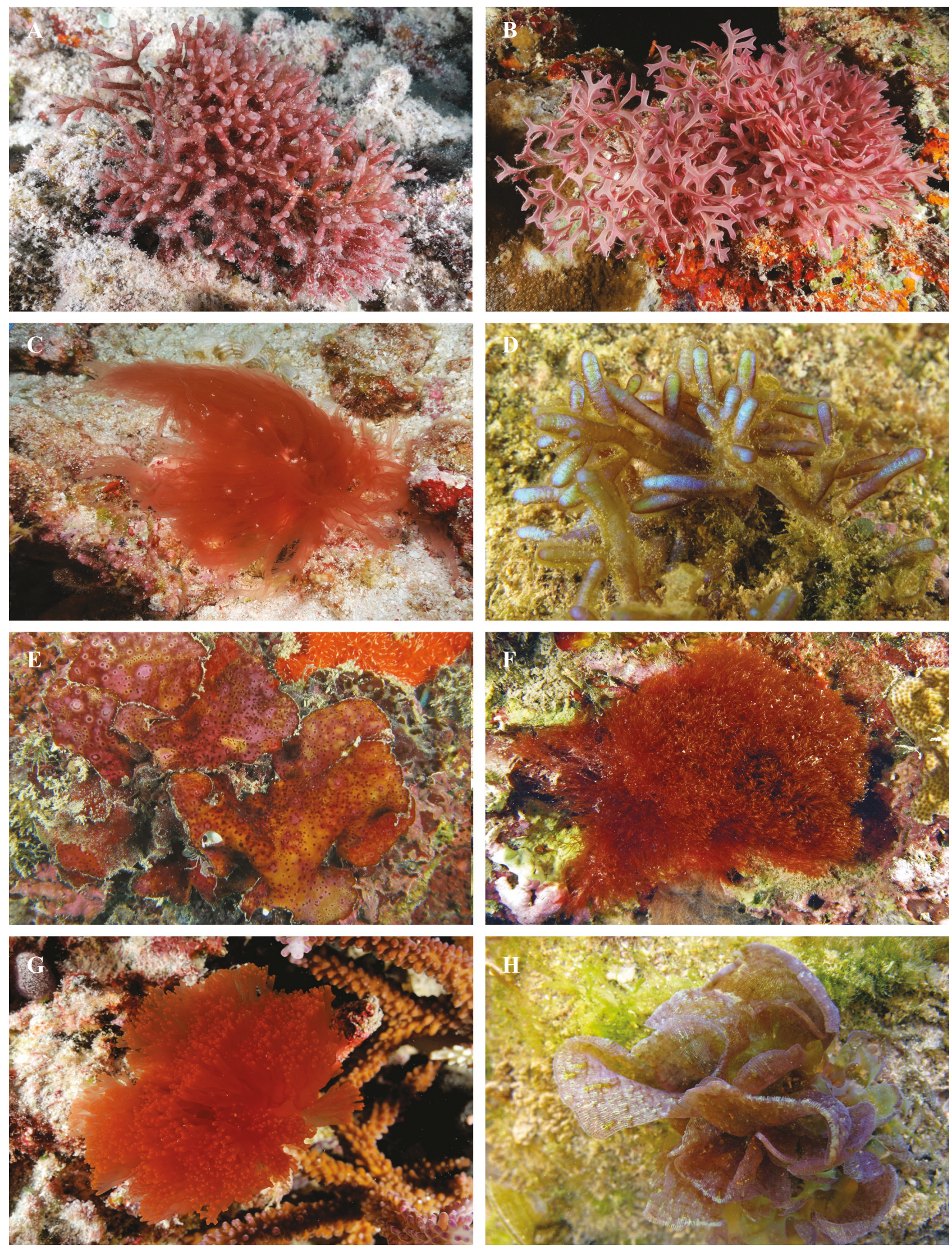

FIGURE 3 Species of red algae from the Kimberley survey area: A) Galaxaura indica (Galaxauraceae); B) Dichotomaria sibogae (Galaxauraceae); C) Acrosymphyton taylorii (Acrosymphytaceae); D) Champia subcompressa (Champiaceae); E) Cryptocallis dixoniorum (Sebdeniaceae); F) Balliella crouanioides (Ceramiaceae); G) Tsengia flammea (Tsengiaceae); H) Martensia millarii (Delesseriaceae). 


\section{DISCUSSION}

The results of the present surveys were incorporated with earlier survey results (e.g., Belton et al. 2014; Huisman 2004; Huisman et al. 2009) into broader studies of the tropical NW Australian marine algal flora, including earlier Kimberley collections and collections from the Pilbara and Gascoyne coasts as far south as Coral Bay (just north of the Tropic of Capricorn). These were presented in two volumes of the 'Algae of Australia' series, the first describing the green and brown algae (Huisman 2015) and the second the red algae (Huisman 2018). Those publications recorded 68 genera and 171 species of green and brown algae and 158 genera and 351 species of red algae. The contents of Huisman (2015) did not include results of the Ashmore and Hibernia Reef survey and the later Rowley Shoals survey, but only two green algal species, Caulerpa filicoides and Tydemania expeditionis, were newly recorded for the region. Both species are relatively common on tropical Pacific reefs, but prior to the 2013 survey had not been recorded for the Western Australian coast.

Huisman and Sampey (2014) collated all historical records of marine plants from the Kimberley and recorded 308 species (88 Chlorophyta, 39 Ochrophyta, 145 Rhodophyta, 12 seagrasses, 18 mangroves and 6 Cyanobacteria). These totals are comparable to those based on our recent surveys, although mangroves and cyanobacteria were not recorded. However, there are notable changes in the species composition between the historical and recent records. These are primarily the result of taxonomic revisions and recognition of new species based on recent collections. Many of the historical records were of species considered to be widespread, but further study on recent specimens, often incorporating DNA sequence analyses, have revealed a greater diversity than previously recognised and a greater number of geographically restricted species. For example, the historical record of the Bahamian red alga Dichotomaria marginata (J. Ellis and Sol.) Lam. has been shown to be misapplied and is now referred to the Indonesian species Dichotomaria sibogae (Weber Bosse) Huisman et al. (Huisman et al. 2018). Similarly, Gibsmithia hawaiiensis Doty is now regarded to be restricted to the Hawaiian Islands and the Kimberley species is referred to Gibsmithia indopacifica Gabriel et al. (Gabriel et al. 2017).

Several of the newly recorded species collected in the recent surveys were originally described from more northern, non-Australian locations, particularly, and not unexpectedly, Indonesia. These species were based on collections made during the Dutch Siboga Expedition (1899-1900) to the Netherlands East Indies [= the Indonesian Archipelago] and described by Anna Weber-van
Bosse. Some 14 species are included in this group, some of which had not been recollected since the original expedition. The recent specimens have enabled a more detailed assessment of the Webervan Bosse taxa, incorporating molecular and morphological analyses. An example is Rhytimenia maculata, which was described originally from Makassar Strait (off Borneo) by Weber-van Bosse (1928) as Kallymenia maculata. Molecular and morphological analyses revealed that this species is incorrectly placed in Kallymenia and the new genus Rhytimenia was erected for the species (Huisman et al. 2016).

The recognition of new species following DNA analyses has also had a significant impact, notably in some of the more 'difficult' groups such as the Peyssonneliaceae, a family of red algae comprising mostly encrusting taxa. The historical records included only a single species, whereas studies of the recently collected material revealed a staggering 17 species in six genera (Dixon, 2018). It is clear these surveys and recent activities have contributed substantially to our understanding of the Kimberley marine flora, but in many cases we have only scratched the surface and further surveys will undoubtedly reveal additional new and unrecorded species.

\section{ACKNOWLEDGEMENTS}

The Western Australian Museum and its partner agencies respectfully acknowledge the Traditional Custodians of the Kimberley land and sea country, of Elders past and present, and in particular the Dambimangari and Wunambal Gaambera peoples, for collections made on their sea country.

We would like to acknowledge and thank the field work organiser Clay Bryce of the Western Australian Museum for his tireless support. Field work was funded by Woodside Energy and the Western Australian Museum and the authors would like to acknowledge their support. Post-survey work was supported by the Australian Biological Resources Study and the Western Australian Department of Biodiversity, Conservation and Attractions.

\section{REFERENCES}

Belton, G.S., Prud'homme van Reine, W.F., Huisman, J.M., Draisma, S.G.A. and Gurgel, C.F.D. (2014). Resolving phenotypic plasticity and species designation in the morphologically challenging Caulerpa racemosa-peltata complex (Chlorophyta, Caulerpaceae). Journal of Phycology 50: 32-54.

Bryce, C., Bryce, M. and Radford, B. (2018a). Project methods and station geomorphology related to a multi-taxon survey (2009-2014) of the Kimberley. Records of the Western Australian Museum Supplement 85: 1-43. doi: 10.18195/issn.0313-122x.85.2018.001-043. 
Bryce, M., Radford, B. and Fabricious, K. (2018b). Soft coral and sea fan (Octocorallia) biodiversity and distribution from a multi-taxon survey (20092014) of the shallow tropical Kimberley, Western Australia. Records of the Western Australian Museum Supplement 85: 45-73. doi: 10.18195/issn.0313122x.85.2018.045-073.

Dixon, K.R. (2018). Peyssonneliales. Algae of Australia: Marine Benthic Algae of North-western Australia, 2. Red Algae. ABRS, Canberra; CSIRO Publishing, Melbourne. pp. 208-244.

Gabriel, D., Draisma, S.G.A., Schmidt, W.E., Schils, T., Sauvage, T., Maridakis, C., Gurgel, C.F.D., Harris, D.J. and Fredericq, S. (2017). Beneath the hairy look: the hidden reproductive diversity of the Gibsmithia hawaiiensis complex (Dumontiaceae, Rhodophyta). Journal of Phycology 53: 1171-1192.

Huisman, J.M. (2004). Marine benthic flora of the Dampier Archipelago, Western Australia. Records of the Western Australian Museum Supplement 66: 61-68. doi: 10.18195/issn.0313-122x.66.2004.061-068.

Huisman, J.M. (2015). Algae of Australia: Marine Benthic Algae of North-western Australia, 1. Green and Brown Algae. ABRS, Canberra; CSIRO Publishing, Melbourne. viii + 320 pp.

Huisman, J.M. (2018). Algae of Australia: Marine Benthic Algae of North-western Australia, 2. Red Algae. ABRS, Canberra; CSIRO Publishing, Melbourne. xii + 672 pp.

Huisman, J.M. (2019). Marine Plants of Australia. Revised Edition. UWA Publishing, xviii + 435 pp.
Huisman, J.M., Boo, G.H., Boo, S.M. and Lin, S.-M. (2018). Galaxauraceae. Algae of Australia: Marine Benthic Algae of North-western Australia, 2. Red Algae. ABRS, Canberra; CSIRO Publishing, Melbourne. pp. 30-49.

Huisman, J.M., Leliaert, F., Verbruggen, H. and Townsend, R.A. (2009). Marine Benthic plants of Western Australia's shelf-edge atolls. Records of the Western Australian Museum Supplement 77: 50-87. doi: 10.18195/issn.0313-122x.77.2009.050-087.

Huisman, J.M. and Sampey, A. (2014). Synthesis of historic marine species data for the Kimberley, Western Australia (1880s-2009): Marine flora. Records of the Western Australian Museum Supplement 84: 45-67. doi: 10.18195/issn.0313-122x.84.2014.045-067.

Huisman, J.M., Saunders, G.W., Le Gall, L. and Vergés, A. (2016). Rhytimenia, a new genus of red algae based on the rare Kallymenia maculata (Kallymeniaceae, Rhodophyta). Phycologia 55: 299-307.

Moore, G.I., Morrison, S.M. and Johnson, J.W. (2020). The distribution of shallow marine fishes of the Kimberley, Western Australia, based on a long-term dataset and multiple methods. Records of the Western Australian Museum Supplement 85: 105-115. doi: 10.18195/ issn.0313-122x.85.2020.105-115.

Weber-van Bosse, A. (1928). Liste des algues du Siboga, IV. Rhodophyceae. Troisème partie. Gigartinales et Rhodymeniales et tableau de la distribution des Chlorophycées, Phaeophycées et Rhodophycées de l'Archipel Malaisien. Siboga-Expeditie Monographie 59d. pp. 393-533, figs 143-213, pls XI-XVI. Leiden: E.J. Brill. 